\title{
Theoretical and experimental screening methods for functional materials design
}

Matthew Dunstan ${ }^{1}$, Cindy Lau ${ }^{2}$, Can Kocer ${ }^{1}$, Wenting $\mathrm{Hu}^{3}$, John Dennis ${ }^{4}$, Andrew Morris ${ }^{5}$, Stuart Scott ${ }^{2}$, Clare Grey ${ }^{1}$ ${ }^{1}$ Department Of Chemistry, University Of Cambridge, Cambridge, United Kingdom, ${ }^{2}$ Department of Engineering, University of Cambridge, Cambridge, United Kingdom, ${ }^{3}$ School of Chemical Engineering and Advanced Materials, Newcastle University, Newcastle, United Kingdom, ${ }^{4}$ Department of Chemical Engineering and Biotechnology, University of Cambridge, Cambridge, United Kingdom, ${ }^{5}$ Department of Physics, University of Cambridge, Cambridge, United Kingdom E-mail: mtd33@cam.ac.uk

With the advent of large structural databases containing both optimised crystallographic structures and their ground state energies, the goal of rationally designing novel functional materials for a variety of applications can be realised. Through selection of relevant, property-specific parameter(s), screening criteria can then be applied to thousands of potential candidates in silico, efficiently selecting the most promising materials for subsequent experimental testing.

Here we describe our work developing screening methodologies for the design of novel materials for carbon capture and storage (CCS) and ionic conductivity. Our holistic approach combines theoretical screening with experimental validation to better understand the trends underpinning the performance of the selected materials. We have made use of the Materials Project database (www.materialsproject.org), which not only contains an extensive variety of calculated structures, but is also constructed in such a way to be amenable to high throughput screening.

The first application we consider is $\mathrm{CO} 2$ absorption looping for CCS, which requires oxide materials that are able to reversibly absorb $\mathrm{CO} 2$ at high temperatures. $\mathrm{CaO}$ is the prototypical material for this application, but unfortunately suffers irreversible capacity loss and sintering upon continuous carbonation-regeneration cycles, necessitating materials with improved performance. With large scale screening, we were able to simulate the carbonation equilibria for 640 prospective sorbents and then select a number of candidates based on (i) minimising the energy cost associated with their use and (ii) maximising their theoretical CO2 capture capacity [1]. The accuracy of the screening was validated using structural and thermogravimetric analysis, and the process led to a number of design rules for optimising materials performance, including focussing on ternary oxides and materials containing magnesium and calcium.

The second part of this work concerns another CCS technology, chemical looping combustion, which utilises materials that can spontaneously release gaseous $\mathrm{O} 2$ in order to efficiently burn fuel in a N2-free environment and create pure $\mathrm{CO} 2$ for subsequent capture. This is in contrast to the former process, which separates $\mathrm{CO} 2$ post-combustion. Binary metal oxides with multiple oxidation states such as $\mathrm{CuO}$ and $\mathrm{MnO} 2$ can be reduced under reactor conditions, but materials that reduce at lower temperatures and cycle more stably are desirable in order to minimise energy costs involved with combustion at higher temperatures. Our screening found over 2200 materials that were able to undergo redox reactions under the specific process conditions [2], with further experimental studies revealing a number of promising materials that could be stably cycled between their perovskite and brownmillerite phases.

The final part details the use of local structural similarity algorithms to efficiently screen materials for oxygen ionic conductivity. Building on a previous methodology using Voronoi tessellations to define a local structural similarity coefficient [3], we characterise the possible conduction networks for oxide anions in close-packed oxide materials found in the Materials Project database, and suggest promising structures for fast ionic conduction.

[1] Dunstan, M. T. et al. (2016). Energy Environ. Sci. 9, 1346-1360.

[2] Lau, C., Dunstan, M. T. et al. (2017). Energy Environ. Sci. DOI: 10.1039/c6ee02763f

[3] Yang, L., Dacek, S. \& Ceder, G. (2014). Phys. Rev. B 90, 054102. 


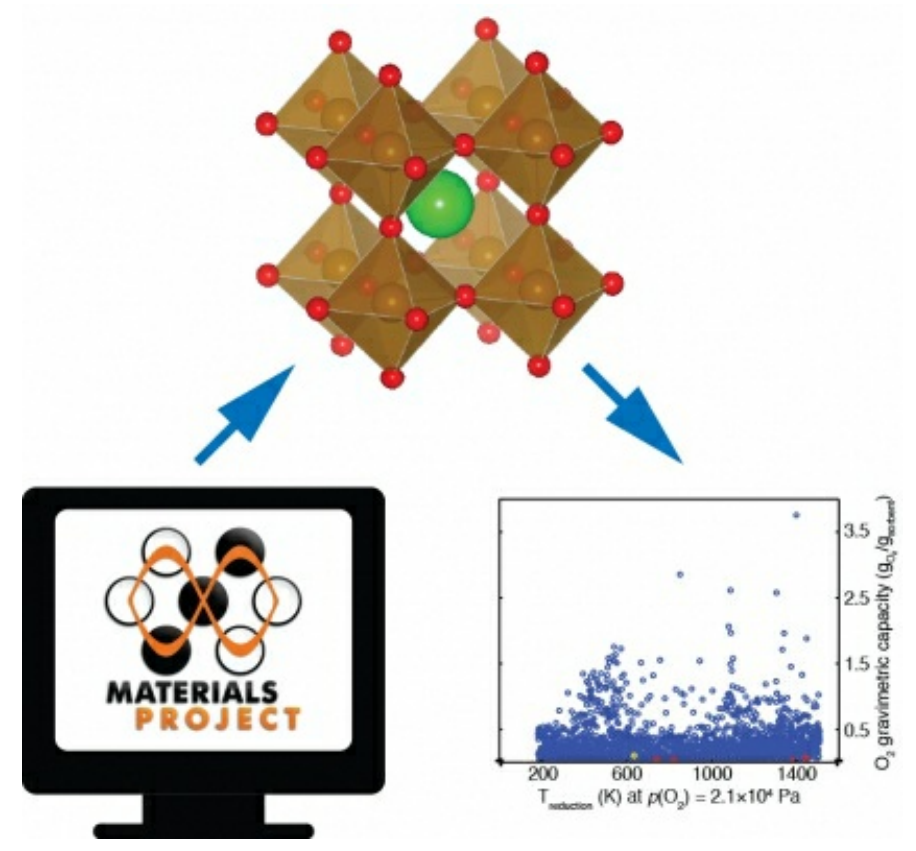

Keywords: CCS, materials discovery, 